Received: 20 February 2019

Accepted: 28 May 2019

Published online: 11 June 2019

\section{Association of antidepressants with brain morphology in early stages of psychosis: an imaging genomics} approach

\author{
Oleg Bykowsky ${ }^{1,2,5}$, Fabienne Harrisberger ${ }^{1,2}$, André Schmidt ${ }^{1,2}$, Renata Smieskova ${ }^{1,2}$, \\ Daniel J. Hauke ${ }^{1,2,4}$, Laura Egloff ${ }^{1,2}$, Anita Riecher-Rössler ${ }^{2}$, Paolo Fusar-Poli ${ }^{3}$, \\ Christian G. Huber $\mathbb{D}^{2}$, Undine E. Lang ${ }^{2}$, Christina Andreou ${ }^{2}$ \& Stefan Borgwardt $\mathbb{D}^{1,2,3}$
}

Depressive symptoms in subjects at Clinical High Risk for Psychosis (CHR-P) or at first-episode psychosis (FEP) are often treated with antidepressants. Our cross-sectional study investigated whether brain morphology is altered by antidepressant medication. High-resolution $\mathrm{T}_{1}$-weighted structural MRI scans of 33 CHR-P and FEP subjects treated with antidepressants, 102 CHR-P and FEP individuals without antidepressant treatment and 55 controls, were automatically segmented using Freesurfer 6.0. Linear mixed-effects modelling was applied to assess the differences in subcortical volume, surface area and cortical thickness in treated, non-treated and healthy subjects, taking into account converted dosages of antidepressants. Increasing antidepressant dose was associated with larger volume of the pallidum and the putamen, and larger surface of the left inferior temporal gyrus. In a pilot subsample of separately studied subjects of known genomic risk loci, we found that in the right postcentral gyrus, the left paracentral lobule and the precentral gyrus antidepressant dose-associated surface increase depended on polygenic schizophrenia-related-risk score. As the reported regions are linked to the symptoms of psychosis, our findings reflect the possible beneficial effects of antidepressant treatment on an emerging psychosis.

Appearance of psychosis is considered to be the driving force of multiple debilitating mental disorders, including schizophrenia $^{1,2}$, which affects $0.7 \%$ of the world population ${ }^{3}$. Psychosis presents itself in a diffuse temporal and phenomenological range of symptoms, of disparate severity and persistence ${ }^{4,5}$. Before their assignation to the first episode of psychosis (FEP) group, subjects at risk belong to a heterogeneous group of Clinical High Risk for Psychosis (CHR-P), characterised by attenuated psychotic symptoms, brief limited intermittent psychotic symptoms, genetic risk or schizotypal traits and a decline in social and occupational functioning ${ }^{6-9}$. The disorder usually manifests itself in early adulthood and the active search for reliable transition markers continues as around $20 \%$ of the CHR-P undergo transition to FEP during the first two years ${ }^{10}$.

Multiple findings indicate that CHR-P and FEP show similar functional ${ }^{11}$ and structural brain abnormalities $^{9,10,12,13}$, but it is unclear to what extent they reflect genetics, general distress, medication effects or are unique features associated with the at risk stage of the disorder. The psychosis vulnerability seems associated with progressive nonlinear morphological changes, to which the pleiotropic genetic factors, the medication and non-genetic factors may contribute ${ }^{14-16}$. CHR-P subjects show structural abnormalities in frontal, cingular and temporal cortices, insular regions, temporal gyrus and some of these changes may be predictive of later transition to psychosis ${ }^{16-24}$.

\footnotetext{
${ }^{1}$ Neuropsychiatry and Brain Imaging, Department of Psychiatry (UPK), University of Basel, Basel, Switzerland. ${ }^{2}$ Psychiatric University Hospital (UPK), University of Basel, Basel, Switzerland. ${ }^{3}$ Early Psychosis: Interventions and Clinical Detection (EPIC) lab, Department of Psychosis Studies, Institute of Psychiatry, Psychology \& Neuroscience, King's College London, London, United Kingdom. ${ }^{4}$ Department of Mathematics and Computer Science, University of Basel, Basel, Switzerland. ${ }^{5}$ Center for Addiction Medicine, Châlons-en-Champagne General Hospital, Châlonsen-Champagne, France. Correspondence and requests for materials should be addressed to S.B. (email: stefan. borgwardt@upkbs.ch)
} 
CHR-P and FEP individuals often suffer from comorbid depressive and anxiety symptoms ${ }^{25,26}$ that can precede ${ }^{27}$ or accompany the onset of attenuated positive psychotic symptoms ${ }^{9,28}$. These features are the main reason for seeking help at specialized services ${ }^{29}$ and the occurrence of these is significantly associated with decreased likelihood of remission from CHR- $\mathrm{P}^{30,31}$. Antipsychotic treatment usually begins after the first episode of psychosis and non-random comparative studies of antidepressants and antipsychotics indicate that antidepressants might be a beneficial effective treatment for preventing psychosis in CHR-P individuals ${ }^{32-34}$.

Currently CHR-P and FEP subjects are occasionally treated with antidepressants if they present depressive symptoms ${ }^{35}$, but the effect of antidepressant medication on brain morphology has, to the best of our knowledge, rarely been explored in the CHR-P and FEP cohorts.

The morphological reduction in major depression was observed in the prefrontal, temporal and parieto-occipital regions, basal ganglia, inferior/middle temporal gyri, hippocampus, and the cerebellum ${ }^{36-39}$. It was shown for depression that antidepressant treatment was connected to enlargement of the frontal cortex, middle frontal gyrus, anterior cingulate, and hippocampus ${ }^{40-44}$. It is linked to an increase in grey matter volume ${ }^{45}$, it influences brain connectivity ${ }^{46}$, and it modulates synaptogenesis, neurogenesis and dendritic arborisation in animal models and in the human hippocampus ${ }^{47-50}$. A recent study of affective psychoses ${ }^{51}$ did not detect long-term antidepressant effect on any brain regions, although - like in other studies - only general presence or absence of antidepressants was used as a predictor.

As the goal of ongoing international research efforts is to establish reliable brain markers to complement clinical features for the prediction of psychosis onset, it is crucial to disentangle disease and medication effects on brain morphology in CHR-P and FEP individuals. Therefore, the aim of our investigation was to study whether morphological features such as cortical thickness, surface area and subcortical volume were altered after administration of antidepressant medication in CHR-P and FEP subjects. The focus on subcortical volumes is based on previous studies in $\mathrm{FEP}^{14,52,53}$ and $\mathrm{UHR}^{54,55}$ patient showing alterations in these cohorts relative to healthy volunteers. Cortical thickness and surface area were further considered because such surface-based measures are probably more sensitive to detect brain alterations in early phases of psychosis ${ }^{56}$.

As there are only a few studies showing an impact of antidepressant medication on volume, thickness and surface $e^{26,51}$ in CHR-P and FEP, we investigated the influence of antidepressant dose on the whole-brain. As shown in the cited literature, antidepressant effect is often linked to modifications in the frontal regions and in hippocampus, and we expected to register the structural effects primarily in these regions.

While in CHR-P and FEP the transition risk and morphology ${ }^{57}$ are co-modulated by genetics, our additional aim was to identify if there is an impact of combined cumulative estimate of known genomic risk loci (polygenic schizophrenia-related risk scores or PSRS ${ }^{58}$ ) on the effect of antidepressants on brain morphology in CHR-P and FEP, as subjects with high/low genetic risk might have different medication effect because of the different illness endophenotypes or a genetic overlap between pathogenesis and drug action ${ }^{59}$. Although a recent study ${ }^{60}$ did not find evidence of genetic overlap between schizophrenia risk and subcortical volume, several studies have already reported an association of a PSRS with brain volume ${ }^{61-63}$. Our focus on analysing the interaction of PSRS and antidepressant medication on thickness, surface and subcortical volume is novel in the context of previous studies and we hypothesise that the antidepressant medication effect on brain morphology may depend on the genetic predisposition for psychosis.

\section{Results}

Demographics and clinical characteristics. Samples with and without genetics data (Table 1) exhibited no significant differences $(p>0.05)$ in age, handedness or IQ. SANS did not differ between CHR-P and FEP. There were significant differences between groups in sex distribution in the full sample $(p<0.001)$, but not in the PSRS subsample $(p>0.05)$, and in the years of education distribution in the full sample $(p<0.001)$, but not in the PSRS subsample $(p>0.05)$. In both samples, there was a significant between-group difference in overall BPRS (both samples $p<0.001)$, cannabis consumption $(p=0.007, p<0.001)$ and $G A F($ both samples $p<0.001)$.

Morphometric differences. We proceed reporting effects that survived FDR correction with a significance threshold of $\mathrm{p}<0.05$ followed by exploratory findings that survived an uncorrected threshold of $\mathrm{p}<0.01$. Table 2 shows a summary of the significant LME results for antidepressants dose effects on brain regions in the whole sample; Table 3 shows the regions for which an interaction between PSRS and medication was required to explain variations in surface or volume.

Subcortical volume. We detected a significant main effect of antidepressant dosage on the putamen $(\beta=0.0166$, s.e. $=0.0053, p=0.0019)$ and the pallidum $(\beta=0.0157$, s.e. $=0.0049, p=0.0016)$, both survived FDR correction. A Tukey post-hoc test revealed an enlargement trend in antidepressant-treated CHR-P and FEP subjects' subcortical volume. The left pallidum in the group of antidepressant-treated CHR-P and FEP subjects was enlarged compared to healthy controls $(p=0.04)$. The putamen was enlarged in antidepressant-treated CHR-P and FEP subjects compared to the non-treated CHR-P and FEP subjects (left $p=0.05$, right $p>0.05$ ) and healthy controls (left $p=0.003$, right $p=0.006$ ).

A group-wise Tukey post-hoc test showed that the medicated CHR-P group exhibited significant enlargement in the left putamen $(p=0.02)$ compared to unmedicated CHR-P and the medicated FEP group exhibited enlargement of the right putamen $(p=0.02)$ and bilateral pallidum (left $p=0.006$, right $p=0.036$ ) compared to the unmedicated FEP.

We also found the main effect of antidepressant dosage on nucleus accumbens (NAcc) $(\beta=0.0152$, s.e. $=0.0051$, $p=0.0033$ ), but it did not survive the FDR correction. A Tukey post-hoc test revealed that left NAcc was enlarged in the group of antidepressant-treated CHR-P and FEP subjects compared to the non-treated CHR-P and FEP subjects $(p=0.02)$. There was a trend to enlargement in left NAcc in medicated FEP group $(p=0.03)$ - compared to the unmedicated group. 


\begin{tabular}{|c|c|c|c|c|c|c|}
\hline & $\begin{array}{l}\text { Combined CHR-P } \\
\text { and FEP without } \\
\text { antidepressants (NT) }\end{array}$ & $\begin{array}{l}\text { Combined CHR-P } \\
\text { and FEP treated with } \\
\text { antidepressants (T) }\end{array}$ & HC & Statistics & P-value & Post-hoc \\
\hline \multicolumn{7}{|c|}{ Number of subjects } \\
\hline PSRS subsample & 43 & 15 & 14 & & & \\
\hline Full sample & 102 & 33 & 55 & & & \\
\hline \multicolumn{7}{|l|}{ Sex M/F } \\
\hline PSRS subsample & '23/10 & $' 12 / 3$ & $6 / 8$ & $\mathrm{X}^{2}=4.43(2)$ & 0.11 & \\
\hline Full sample & $71 / 31$ & $23 / 10$ & '22/33 & $\mathrm{X}^{2}=14.2(2)$ & 0.0008 & \\
\hline \multicolumn{7}{|c|}{ Mean age in years (s.d.) } \\
\hline PSRS subsample & $24.58(6)$ & $26.87(7.23)$ & $26.4(2.75)$ & Anova $\mathrm{F}$ value $=1(2)$ & 0.37 & \\
\hline Full sample & $25.29(6.13)$ & $26.55(6.56)$ & $25.93(4.68)$ & Anova F value $=0.64(2)$ & 0.53 & \\
\hline \multicolumn{7}{|c|}{ Handedness left/non-left } \\
\hline PSRS subsample & $6 / 37$ & $2 / 13$ & ' $1 / 13$ & $\mathrm{X}^{2}=0.516(2)$ & 0.77 & \\
\hline Full sample & $' 10 / 91$ & $2 / 31$ & $3 / 35$ & $\mathrm{X}^{2}=0.306(2)$ & 0.86 & \\
\hline \multicolumn{7}{|c|}{ Years of education (s.d.) } \\
\hline PSRS subsample & $13.58(2.6)$ & $14.33(3.36)$ & $16.1(3.4)$ & Anova F value $=2.08(2)$ & 0.13 & \\
\hline Full sample & $13.23(2.6)$ & $14.24(3.23)$ & $15.32(2.76)$ & Anova F value $=8.22(2)$ & 0.0004 & $\mathrm{HC}>\mathrm{NT}$ \\
\hline \multicolumn{7}{|l|}{ IQ (s.d.) } \\
\hline PSRS subsample & $108.70(14.53)$ & $115.33(19.18)$ & $117.6(11.2)$ & Anova F value $=1.99(2)$ & 0.15 & \\
\hline Full sample & $108.44(15.02)$ & $113.79(16.15)$ & $116.5(11.43)$ & Anova F value $=2.87(2)$ & 0.061 & \\
\hline \multicolumn{7}{|l|}{ BPRS (s.d.) } \\
\hline PSRS subsample & $45.46(15.17)$ & $41(10.91)$ & $24.4(0.91)$ & Anova F value $=14.4(2)$ & $<0.0001$ & $\mathrm{HC}<\mathrm{T}$, NT \\
\hline Full sample & $42.63(13.57)$ & $40.67(9.09)$ & $24.16(0.57)$ & Anova F value $=56.1(2)$ & $<0.0001$ & $\mathrm{HC}<\mathrm{T}$, NT \\
\hline \multicolumn{7}{|c|}{ BPRS affective (s.d.) } \\
\hline PSRS subsample & $6.54(3.44)$ & $7.07(2.53)$ & $3.20(0.56)$ & Anova $\mathrm{F}$ value $=8.44(2)$ & 0.0006 & $\mathrm{HC}<\mathrm{T}$, NT \\
\hline Full sample & $6.02(2.90)$ & $7.03(2.61)$ & $3.09(0.35)$ & Anova F value $=36.6(2)$ & $<0.0001$ & $\mathrm{HC}<\mathrm{T}$, NT \\
\hline \multicolumn{7}{|c|}{ BPRS negative (s.d.) } \\
\hline PSRS subsample & $6.15(3.07)$ & $6.21(2.64)$ & $3(0)$ & Anova F value $=7.93(2)$ & 0.0008 & $\mathrm{HC}<\mathrm{T}$, NT \\
\hline Full sample & $5.25(2.81)$ & $6.23(2.66)$ & $3(0)$ & Anova F value $=24.3(2)$ & $<0.0001$ & $\mathrm{HC}<\mathrm{T}$, NT \\
\hline \multicolumn{7}{|c|}{ BPRS positive (s.d.) } \\
\hline PSRS subsample & $8.56(4.52)$ & $7.21(4.37)$ & $3(0)$ & Anova F value $=9.95(2)$ & 0.0002 & $\mathrm{HC}<\mathrm{T}$, NT \\
\hline Full sample & $8.07(4.52)$ & $6.80(3.63)$ & $3(0)$ & Anova F value $=34.7(2)$ & $<0.0001$ & $\mathrm{HC}<\mathrm{T}, \mathrm{NT}$ \\
\hline \multicolumn{7}{|c|}{ BPRS activation (s.d.) } \\
\hline PSRS subsample & $3.85(1.87)$ & $3(0.78)$ & $3(0)$ & Anova F value $=2.65(2)$ & 0.079 & \\
\hline Full sample & $3.85(1.92)$ & $3.23(0.97)$ & $3(0)$ & Anova $\mathrm{F}$ value $=6.43(2)$ & 0.002 & $\mathrm{HC}<\mathrm{NT}$ \\
\hline \multicolumn{7}{|l|}{ SANS (s.d.) } \\
\hline PSRS subsample & $17.61(14.38)$ & $18.71(12.53)$ & NA & $\mathrm{t}=1.4$ & 0.2 & \\
\hline Full sample & $14.10(12.64)$ & $17.70(12.42)$ & & $\mathrm{t}=0.27$ & 0.8 & \\
\hline \multicolumn{7}{|l|}{ GAF (s.d.) } \\
\hline PSRS subsample & $64.81(17.32)$ & $63.93(13.14)$ & $89.3(4.9)$ & Anova F value $=15.8(2)$ & $<0.0001$ & $\mathrm{HC}>\mathrm{T}$, NT \\
\hline Full sample & 65.11(15.96) & $60.81(13.16)$ & $91.9(4.5)$ & Anova F value $=89(2)$ & $<0.0001$ & $\mathrm{HC}>\mathrm{T}$, NT \\
\hline \multicolumn{7}{|c|}{ Antipsychotics no/yes } \\
\hline PSRS subsample & $35 / 8$ & $11 / 4$ & NA & $\mathrm{X}^{2}=0.086(1)$ & 0.08 & \\
\hline Full sample & $73 / 29$ & $24 / 9$ & & $\mathrm{X}^{2} \sim 0(1)$ & $\sim 1$ & \\
\hline \multicolumn{7}{|c|}{ Cannabis no/yes/NA } \\
\hline PSRS subsample & $34 / 9 / 0$ & $6 / 8 / 1$ & $13 / 1 / 0$ & $\mathrm{X}^{2}=9.95(2)$ & 0.0069 & \\
\hline Full sample & $80 / 21 / 1$ & $17 / 13 / 3$ & $52 / 2 / 1$ & $\mathrm{X}^{2}=20.9(2)$ & $<0.0001$ & \\
\hline \multicolumn{7}{|c|}{ Normalized PSRS (s.d.) } \\
\hline PSRS subsample & $0.02(1)$ & $0.02(1.05)$ & & Anova F value $=0.09(2)$ & 0.92 & \\
\hline
\end{tabular}

Table 1. Demographics and clinical characteristics. sd: standard deviation; T: treated by antidepressants; NT: not treated by antidepressants; NA: no data; all post-hoc analyses correspond to $\mathrm{p}<0.05$.

We found no significant interaction effects of antidepressant dosage and PSRS for subcortical structure volume.

Surface area. We detected a significant main effect of antidepressant dosage on the surface of the inferior temporal gyrus $(\beta=0.0154$, s.e. $=0.0048, p=0.0014$, survived FDR $)$. For the surface of the left inferior temporal gyrus the Tukey post-hoc test revealed the significant enlargement $(p=0.01)$ in antidepressant-treated CHR-P and FEP subjects compared to non-treated CHR-P and FEP subjects. 
We detected a significant main effect of antidepressant dosage on the surfaces of the fusiform gyrus $(\beta=0.0134$, s.e. $=0.0049, p=0.0067)$, the middle temporal gyrus $(M T G)(\beta=0.0151$, s.e $=0.0054, p=0.0055)$, and the inferior parietal lobule $(\beta=0.0152$, s.e. $=0.0053, p=0.005)$ and they did not survive the FDR correction threshold.

The Tukey post-hoc test revealed the significant enlargements in antidepressant-treated CHR-P and FEP subjects compared to non-treated CHR-P and FEP subjects in the surface of the left fusiform gyrus $(p=0.01)$ and the right inferior parietal lobule $(p=0.048)$.

A group-wise Tukey post-hoc test registered the significant $(p=0.01)$ increase in the surface of the medicated FEP group compared to the non-medicated FEP group in a right inferior parietal lobule and the non significant $(p>0.05)$ trend to increase in the left fusiform gyrus, the left inferior temporal gyrus, the left inferior parietal lobule.

We found a significant interaction effect of antidepressant dosage and PSRS (Table 3) on the surface of the precentral gyrus $(p=0.0023)$, the postcentral gyrus $(p=0.0028)$, the paracentral lobule $(p=0.0014)$ and the lateral orbitofrontal cortex $(p=0.0095)$. All interactions except in the lateral orbitofrontal cortex survived the FDR correction in 2 groups LME.

Statistical comparison of the slopes of the LME lines (Figs 1-4) by a Tukey post-hoc test showed that there was a significant increase in the surface of the bilateral precentral gyrus (left and right $p=0.05$; Fig. 1), right postcentral gyrus ( $p=0.004$; Fig. 2$)$ and left paracentral lobule ( $p=0.04$; Fig. 3$)$ and a non-significant increase trend in the lateral orbitofrontal cortex ( $p>0.05$; Fig. 4 ) in antidepressant-treated CHR-P and FEP subjects with increasing PSRS and current dose of antidepressants, but a slight decrease in non-treated CHR-P and FEP subjects.

Cortical thickness. We found no significant main effects of antidepressant dosage on the cortical thickness of the analysed regions nor an interaction effect between antidepressant dosage and PSRS.

LR tests. The LR tests (Tables S4 and S5) showed that the difference between the model pairs were significant $(p<0.01)$ for all models reported in Tables 2 and 3, on the supposition that the antidepressant medication effect in the full sample and the interactions between PSRS and medication in the subsample were significant to explain variation in the data. The same test with 2 groups (CHR-P and FEP) excluding healthy controls confirmed our results for all models.

Correlation analysis. The correlation analysis between the surface and volume of brain regions and the BPRS, SANS and GAF in the full sample and in the PSRS subsample (Tables S2 and S3) showed the negative correlations ( $p<0.05$ uncorrected) in combined CHR-P and FEP subjects for left fusiform gyrus surface and BPRS-activation symptom subscale $(r=-0.207)$, left inferior temporal gyrus surface and BPRS $(r=-0.251)$, BPRS-activation $(r=-0.22)$ and BPRS-positive $(r=-0.226)$, left middle temporal gyrus surface with BPRS $(r=-0.232)$ and BPRS-positive $(r=-0.235)$, left lateral OFC surface with BPRS $(r=-0.309)$, and with SANS $(r=-0.283)$, left paracentral lobule surface and SANS $(r=-0.274)$; right inferior temporal gyrus surface with BPRS-affective $(r=-0.176)$, right MTG surface with BPRS-activation $(r=-0.199)$, right lateral OFC surface with BPRS $(r=-0.265)$, BPRS-positive $(r=-0.335)$ and with GAF $(r=0.396)$, right precentral gyrus surface with BPRS-affective $(r=-0.327)$. The left pallidum volume was positively correlated with BPRS-negative subscale $(r=0.238)$ and with SANS $(r=0.223)$.

\section{Discussion}

In an analysis of automatically segmented brain regions in CHR-P, FEP and healthy controls, we found a significant (FDR p $<0.05$ ) main effect of current antidepressant dose on the volume of the pallidum and the putamen and surface of the inferior temporal gyrus. Furthermore, we found (FDR p $<0.05)$ that the surface of the postcentral gyrus, the paracentral lobule and the precentral gyrus can be linked to an interaction between antidepressants dosage and genomic risk score.

The volumes of the bilateral putamen and the left pallidum were enlarged in antidepressant-treated CHR-P and FEP compared to the untreated cohort and healthy controls. Diagnosis-related increases in the volume of pallidum and the putamen have been previously reported for schizophrenia and FEP patients ${ }^{57,64,65}$. Putamen and pallidum volume have been identified as predictors of positive symptoms and the duration of illness ${ }^{52,64}$ and it was shown that putamen lesions may lead to psychosis ${ }^{66}$. Moreover, the basal ganglia system has been associated with the dopaminergic hypothesis of schizophrenia ${ }^{2}$.

We found a significant main effect of antidepressant dose on the surface of the inferior temporal gyrus. In particular, the antidepressant-treated CHR-P and FEP subjects exhibited enlarged surface of the left inferior temporal gyrus compared to the non-treated CHR-P and FEP subjects. In patients with psychosis a decreased volume ${ }^{67-71}$ and surface area $^{72}$ in the inferior temporal gyrus was demonstrated. Therefore, our finding suggests that antidepressant treatment might increase surface area of the inferior temporal gyrus in early stages of psychosis.

In the subsample with calculated PSRS, we found an interaction effect between antidepressant dose and PSRS on the surface of the precentral and the postcentral gyri, and the paracentral lobule. With the rise in both antidepressant dosage and PSRS, the bilateral precentral gyrus, the right postcentral gyrus and the left paracentral lobule increased in surface - compared to non-treated CHR-P and FEP subjects, where a decreasing trend appeared.

Morphological and functional changes in these regions have been previously associated with psychosis: reduced surface area in the right postcentral gyrus have been reported in 22q11DS subjects ${ }^{73}$ and patients with schizophrenia revealed significant reduction in surface area in frontal and parietal regions $;{ }^{74}$ the displacement ${ }^{75}$, abnormal activation ${ }^{76}$ and the reduction ${ }^{77,78}$ of the postcentral gyrus are characteristic for psychosis.

The regions where the increase in PSRS is linked to the surface increase due to antidepressant treatment are connected to important clinical symptoms. The precentral gyrus has been associated with verbal hallucinations ${ }^{79}$, neurocognitive deficits and attentional deficits ${ }^{80}$. Connectivity changes in the postcentral gyrus with the dorsolateral prefrontal cortex are linked to the improvement in affective psychotic symptoms ${ }^{81}$. 


\begin{tabular}{|c|c|c|c|c|c|c|c|c|c|c|c|c|c|c|}
\hline & \multicolumn{2}{|c|}{$\begin{array}{l}\text { Fusiform gyr. } \\
\text { surf. }\end{array}$} & \multicolumn{2}{|l|}{ GP vol. } & \multicolumn{2}{|c|}{$\begin{array}{l}\text { Inferior parietal } \\
\text { lob. surf. }\end{array}$} & \multicolumn{2}{|c|}{$\begin{array}{l}\text { Inferior temporal } \\
\text { gyr. surf. }\end{array}$} & \multicolumn{2}{|c|}{ MTG surf. } & \multicolumn{2}{|c|}{ NAcc vol. } & \multicolumn{2}{|c|}{ Putamen vol. } \\
\hline & F & P-value & F & P-value & F & P-value & F & P-value & F & P-value & F & $P$-value & F & P-value \\
\hline \multicolumn{15}{|l|}{ FEP, CHR-P, HC } \\
\hline Age & 0.0051 & 0.9434 & 0.0053 & 0.942 & 0.8376 & 0.3613 & 2.3312 & 0.1285 & 7.1333 & $0.0082^{* * * *}$ & 2.733 & 0.1 & 0.724 & 0.3959 \\
\hline Antidepressants dose & 7.5094 & $0.0067^{*}$ & 10.2445 & $0.0016 * * *$ & 8.0283 & $0.0051^{*}$ & 10.4924 & $0.0014 * * *$ & 7.8861 & $0.0055^{*}$ & 8.8841 & 0.0033* & 9.9351 & 0.0019 *** \\
\hline $\begin{array}{l}\text { Antidepressants dose } \mathrm{x} \\
\text { hemisphere }\end{array}$ & 0.9359 & 0.3346 & 0.1058 & 0.7454 & 1.9489 & 0.1644 & 1.0673 & 0.3029 & 0.0466 & 0.8294 & 0.2529 & 0.6156 & 0.5055 & 0.478 \\
\hline Diagnosis & 3.7807 & 0.0246 & 2.2706 & 0.1061 & 0.0022 & 0.9978 & 5.1127 & 0.0069* & 0.1802 & 0.8352 & 2.2488 & 0.1084 & 2.4659 & 0.0877 \\
\hline Hemisphere & 0.017 & 0.8965 & 0.4368 & 0.5095 & 0.0481 & 0.8266 & 0.0013 & 0.9716 & 0.67 & 0.4142 & 0.0015 & 0.9697 & 0.0908 & 0.7635 \\
\hline Sex & 5.1986 & 0.0237 & 9.8685 & $0.002 * * *$ & 1.1203 & 0.2912 & 4.0807 & 0.0448 & 1.1284 & 0.2895 & 1.0903 & 0.2978 & 6.8627 & $0.0095 * * *$ \\
\hline \multicolumn{15}{|l|}{ FEP, CHR-P } \\
\hline Age & 1.2241 & 0.2706 & 0.4671 & 0.4956 & 0.0062 & \begin{tabular}{|l|}
0.9376 \\
\end{tabular} & 0.085 & 0.7711 & 4.1447 & 0.0438 & 1.6135 & 0.2063 & 0.1921 & 0.6619 \\
\hline Antidepressants dose & 7.0187 & $0.0091^{*}$ & 10.8786 & $0.0013 * * *$ & 7.2448 & $0.008^{*}$ & 9.3836 & $0.0027^{*}$ & 7.2142 & $0.0082^{*}$ & 8.0509 & $0.0053^{*}$ & 11.2135 & $0.0011 * * *$ \\
\hline $\begin{array}{l}\text { Antidepressants dose } \mathrm{x} \\
\text { hemisphere }\end{array}$ & 0.9548 & 0.3303 & 0.3089 & 0.5793 & 2.1568 & 0.1443 & 1.378 & 0.2426 & 0.1566 & 0.693 & 0.0884 & 0.7667 & 0.4115 & 0.5223 \\
\hline Diagnosis & 3.2054 & 0.0757 & 4.2515 & 0.0412 & 0.0094 & 0.9228 & 9.2156 & $0.0029 *$ & 0.1988 & 0.6564 & 1.3446 & 0.2484 & 2.4243 & 0.1219 \\
\hline Hemisphere & 0.0104 & 0.9188 & 0.0043 & 0.9478 & 0.1529 & 0.6964 & 0.0797 & 0.7781 & 1.0156 & 0.3156 & 0.1842 & 0.6685 & 0.0027 & 0.9588 \\
\hline Sex & 4.3878 & 0.0381 & 13.6249 & $0.0003^{* * *}$ & 3.3 & 0.0716 & 3.1462 & 0.0785 & 0.4173 & 0.5195 & 1.3707 & 0.2438 & 12.7727 & $0.0005^{* * *}$ \\
\hline
\end{tabular}

Table 2. Summarized linear mixed effects models for antidepressants. *P-value $<0.01, * * *$ survived correction for FDR with P-value < 0.05, GP: Globus Pallidus, MTG: medial temporal gyrus, NAcc: nucleus accumbens, gyr.: gyrus, lob.: lobule, surf: surface, vol.: volume.

\begin{tabular}{|c|c|c|c|c|c|c|c|c|}
\hline & \multicolumn{2}{|c|}{ Lat. OFC surf. } & \multicolumn{2}{|c|}{ Paracentral lob. surf. } & \multicolumn{2}{|c|}{ Postcentral gyr. surf. } & \multicolumn{2}{|c|}{ Precentral gyr. surf. } \\
\hline & F & P-value & F & P-value & F & P-value & F & P-value \\
\hline \multicolumn{9}{|l|}{ FEP, CHR-P, HC with PSRS } \\
\hline Age & 4.6873 & 0.0341 & 0.869 & 0.3548 & 3.3973 & 0.07 & 0.517 & 0.4748 \\
\hline ADeq $x$ PSRS & 7.0471 & $0.0095^{*}$ & 10.8924 & $0.0014^{*}$ & 9.3919 & $0.0028^{*}$ & 9.848 & $0.0023^{*}$ \\
\hline ADeq $x$ PSRS $x$ hemisphere & 4.4517 & 0.0384 & 2.5105 & 0.1177 & 0.2056 & 0.6517 & 0.0081 & 0.9286 \\
\hline Antidepressants dose & 2.9211 & 0.0923 & 3.5752 & 0.0632 & 4.08 & 0.0477 & 4.7202 & 0.0335 \\
\hline Diagnosis & 0.0452 & 0.9559 & 0.5532 & 0.5778 & 0.2971 & 0.744 & 0.5067 & 0.6049 \\
\hline Hemisphere & 0.1085 & 0.7428 & 0.2191 & 0.6412 & 0.0045 & 0.9466 & 0.1297 & 0.7198 \\
\hline PSRS & 0.0187 & 0.8917 & 0.1503 & 0.6996 & 0.5883 & 0.4459 & 0.474 & 0.4937 \\
\hline Sex & 1.1951 & 0.2784 & 0.0795 & 0.7788 & 0.0593 & 0.8085 & 0 & 0.9999 \\
\hline \multicolumn{9}{|l|}{ FEP and CHR-P with PSRS } \\
\hline Age & 3.8094 & 0.0565 & 0.6519 & 0.4232 & 4.1523 & 0.0468 & 0.4273 & 0.5163 \\
\hline ADeq x PSRS & 7.0548 & 0.0098* & 10.3231 & $0.002 * * *$ & 11.4315 & $0.0011 * * *$ & 10.5822 & $0.0017 * * *$ \\
\hline ADeq $x$ PSRS $x$ hemisphere & 4.2635 & 0.0436 & 2.4821 & 0.121 & 0.1726 & 0.6795 & 0.0039 & 0.9504 \\
\hline Antidepressants dose & 2.8239 & 0.099 & 3.0493 & 0.0869 & 4.1067 & 0.048 & 4.1415 & 0.0471 \\
\hline Diagnosis & 0.0009 & 0.9765 & 0.6933 & 0.4089 & 0.0878 & 0.7681 & 0.5546 & 0.4599 \\
\hline Hemisphere & 0.0433 & 0.8359 & 0.1112 & 0.7401 & 0.0638 & 0.8015 & 0.0194 & 0.8896 \\
\hline PSRS & 0.1816 & 0.6718 & 0.2093 & 0.6493 & 1.8401 & 0.1808 & 1.5295 & 0.2219 \\
\hline Sex & 0.6018 & 0.4415 & 0.0055 & 0.9412 & 1.0283 & 0.3154 & 0.1753 & 0.6772 \\
\hline
\end{tabular}

Table 3. Summarized linear mixed effects models for antidepressants $x$ PSRS interaction in the PSRS subsample. *P-value $<0.01$, ***survived correction for FDR with P-value $<0.05$, OFC: orbito-frontal cortex, lat.: lateral, surf: surface, vol.: volume, ADeq: antidepressant medication fluoxetine equivalents, PSRS: polygenic schizophrenia risk score.

According to our findings, one could suggest that antidepressant medication might have a stronger influence on surfaces than thicknesses. Their relation is contradictory, as there is evidence that they're negatively correlated ${ }^{82}$, but also that they're genetically unrelated and develop independently ${ }^{83}$. This is in agreement with our finding that only surface but not thickness shows an interaction between antidepressants and PSRS. So the effect of antidepressants might be genetically determined and only evident for surfaces. Future research is warranted whether the effect of antidepressants in FEP/CHR-P is specific for surfaces and whether it is determined by genetic predisposition.

The overall improvements in CHR-P and schizophrenia after antidepressant treatment reported in the literature are quite inconsistent ${ }^{84,85}$. According to the study of remission from major depression ${ }^{86}$, remitters show increase over time and nonremitters show decrease in volume and thickness of cortical and subcortical structures.

The relationship between antidepressant dosage and morphology identified in our study should be further examined to determine whether antidepressants can efficiently alleviate symptoms and which dose is required; 


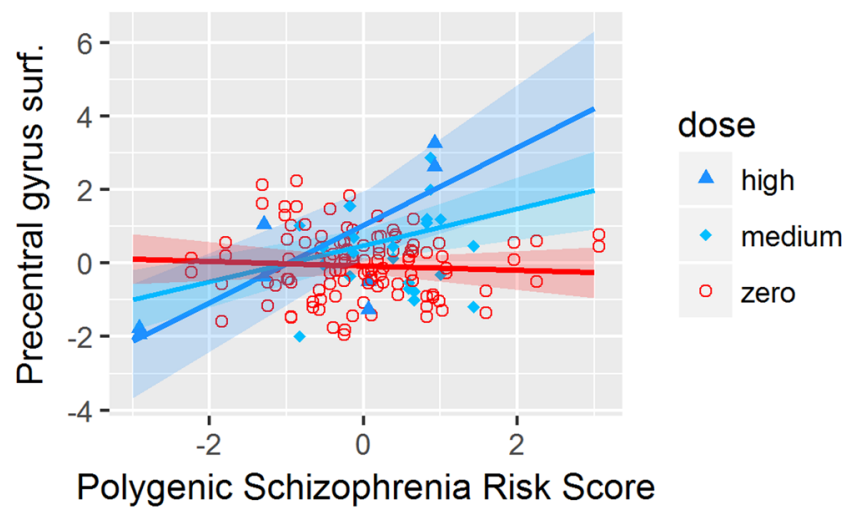

Figure 1. LME for significant interaction between antidepressants dosage and genomic risk score for the surface of the precentral gyrus.

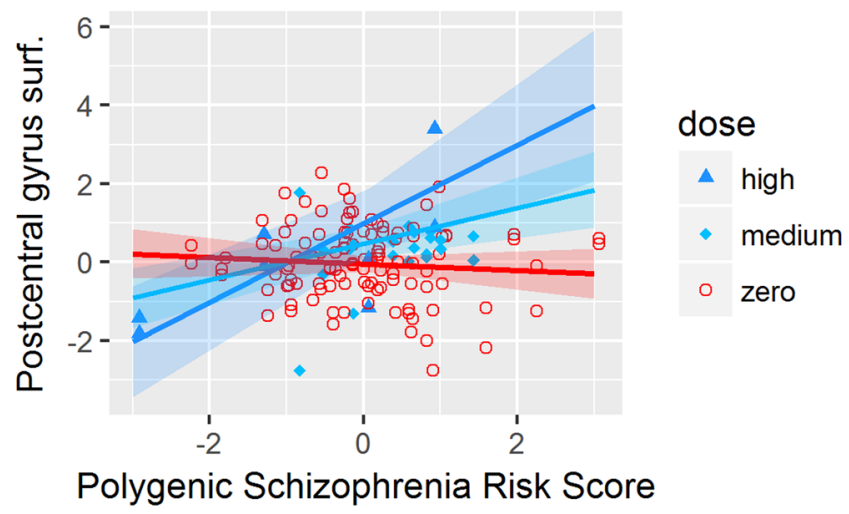

Figure 2. LME for significant interaction between antidepressants dosage and genomic risk score for the surface of the postcentral gyrus.

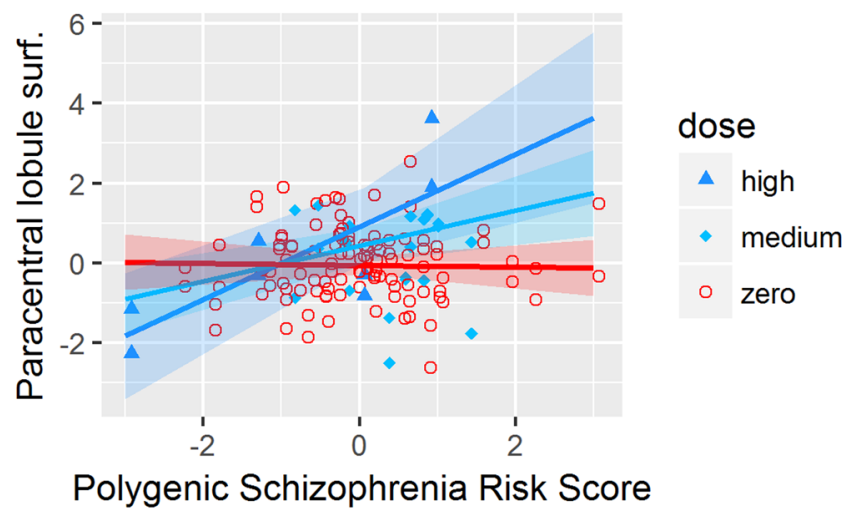

Figure 3. LME for significant interaction between antidepressants dosage and genomic risk score for the surface of the paracentral lobule.

some efforts are already ongoing ${ }^{87}$. Thus, determination of the dosage, start of prescription and combination with other drugs might be improved in subjects with emerging psychosis.

In further studies we consider using the duration of antidepressant admission because we cannot exclude a possibility that those receiving antidepressants may represent a different subpopulation.

The evidence of antidepressant effects on the morphophysiology of regions that have a possible link to clinical symptoms may provide a scientific rationale to support the notion that earlier antidepressant prescription for CHR-P patients could improve their clinical outcomes ${ }^{34}$. As the regional enlargements are negatively correlated to symptoms, the interactions between PSRS and antidepressant dose found in our pilot study of the PSRS subsample might also be of clinical interest as we could speculatively propose that subjects with higher PSRS may benefit 


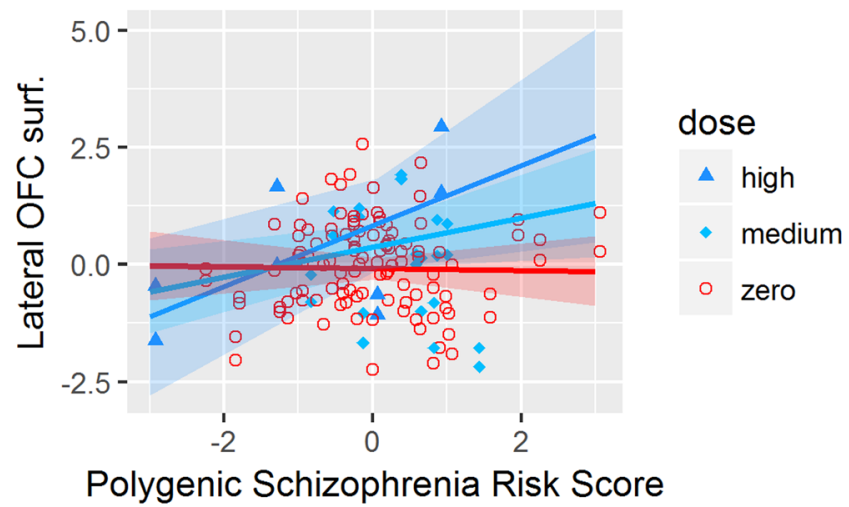

Figure 4. LME for significant interaction between antidepressants dosage and genomic risk score for the surface of the lateral orbitofrontal cortex.

more from antidepressant treatment in order to reduce early symptoms and to limit the morphophysiological side effects. Thus, the translational significance of the findings is that these associations may influence the clinical choice of optimal medication in CHR-P and FEP subjects.

Our study had some limitations. Differences in cannabis consumption among groups may also have been influenced our findings, although the effect of cannabis on morphological brain measures in healthy volunteers and psychotic patients is inconclusive ${ }^{88-91}$. It was not possible to calculate the cumulative lifetime treatment dose of antidepressants due to gaps in medical records. Nevertheless, further studies should include the lifetime dose, as mean dose provides incomplete information in cross-sectional paradigms. The mean antidepressant dose used in the clinic is relatively low and due to the presence of agents with a mixed range of pharmacological action and the limited sample size, we were unable to differentiate groups based on antidepressant types. Our sample size did not allow a meaningful statistical subanalysis to differentiate between the different clinical high-risk subgroups APS, BLIPS and GRD $^{9}$. Given that their risk of developing psychosis is different ${ }^{13}$, future studies are advised to stratify their findings across these subgroups. The number of tests was large due to the multitude of regions possibly implicated in response to antidepressant treatment. The current inferences about the PSRS effect are speculative because of the modest sample, and the future studies should investigate different morphophysiological effects in low and high PSRS cohort, as more genetic data should be collected. The effect of disease stage should also be included in the larger sample. The underlying biological mechanism of these modifications needs to be further investigated.

In sum, to our knowledge, we have demonstrated for the first time the association of the converted antidepressant dosage on morphological brain changes and the interaction between antidepressants dosage and genomic risk score in CHR-P and FEP subjects. As most of the reported regions were shown as linked to the clinical symptoms of psychosis, our findings may contribute to explanations of the suggested beneficial effects of antidepressant treatment in this population.

\section{Methods}

Participants. We use the cross-sectional data of 142 patients (72 FEP, 70 CHR-P) and 55 controls recruited in the FePsy (early detection of psychosis) study (see full Participants, Screening and Genotyping description in Supplementary materials). The PSRS data was obtained for a subsample of 61 patients: 32 FEP and 29 CHR-P, as well as for 14 controls. The data overlaps $(n=72)$ with our previous studies ${ }^{57}$.

All participants provided written informed consent and received compensation for participating. The studies had permission from the ethics committee beider Basel (EKBB). All methods were performed in accordance with the relevant guidelines and regulations.

Screening. The participants were assessed using the Basel Screening Instrument for Psychosis (BSIP) ${ }^{92}$. Subjects selected for the study by screening subsequently underwent an entry examination, which included the Brief Psychiatric Rating Scale (BPRS), Scale for the Assessment of Negative Symptoms (SANS), and a neuropsychological test battery. Inclusion criteria in the CHR-P group required (a) attenuated symptoms or (b) brief limited intermittent psychotic symptoms and genetic risk or schizotypal features, coupled with functional deterioration $^{93}$. The transition to FEP required the occurrence of at least one positive psychotic symptom several times a week for a continuous period of time.

Exclusion criteria. Age below 18 years, insufficient knowledge of German, IQ $<70$, previous episode of psychosis treated with major tranquillisers for $>3$ weeks, a psychosis due to organic reasons or substance abuse, or psychotic symptomatology within a clearly diagnosed affective psychosis or borderline personality disorder.

Medication. The current mean converted antidepressants dose was $28.14 \mathrm{mg}$ Fluoxetine equivalent ${ }^{94}$ in the full sample and $29.53 \mathrm{mg}$ for the PSRS subsample. The assignment of antidepressant treatment in our sample was non-random i.e. according to clinical needs and consisted of escitalopram $(n=10)$, fluoxetine $(n=4)$, citalopram $(\mathrm{n}=2)$, paroxetine $(\mathrm{n}=2)$, sertraline $(\mathrm{n}=2)$; venlafaxine $(\mathrm{n}=4)$; mirtazapine $(\mathrm{n}=5)$; trazodone $(\mathrm{n}=2)$ and bupropion $(n=1)$. The PSRS subsample included escitalopram $(n=3)$, fluoxetine $(n=4)$, sertraline $(n=1)$; venlafaxine 
$(\mathrm{n}=4)$; mirtazapine $(\mathrm{n}=2)$ and trazodone $(\mathrm{n}=2)$ treatment. $\mathrm{N}=38$ subjects from the full sample and $\mathrm{n}=12$ subjects from PSRS subsample were medicated with second-generation antipsychotics (SGA). Current antipsychotic dose was converted into chlorpromazine (CPZ) equivalents ${ }^{95}$. The mean CPZ equivalents (s.d.) were 214.5 (271.1). $\mathrm{N}=9$ subjects were taking both SGA and antidepressants. No healthy control was medicated with antidepressants or SGA. $\mathrm{N}=36$ subjects from the full sample and $\mathrm{n}=18$ subjects from PSRS subsample consumed cannabis.

Genotyping and PSRS calculation. DNA in the PSRS subsample was extracted from whole-blood samples. PSRS was calculated by taking linkage disequilibrium-pruned loci ${ }^{58,96}$. A total of 87 SNPs that could be mapped to one of the top SNPs of the 108 loci associated with schizophrenia and that survived quality control were used to calculate the PSRS. The number of risk alleles per person was weighted for each SNP by the logarithm of its odds ratio as reported in the PGC SZ data set and summed across SNPs ${ }^{97}$. The PSRS was then corrected for the first 20 genotypic principal components (PCs) and the number of SNPs used to calculate the PSRS by using the $\mathrm{z}$-transformed residuals of a linear regression.

Acquisition and analysis of MRI data. We obtained structural MRI scans within an average of 25 days after entry into our early detection service, using a 3 T MR imaging scanner (Siemens Magnetom Verio, Siemens Healthcare, Erlangen, Germany) with a 12-channel phased-array radio frequency head coil. For structural images, a $3 \mathrm{D} \mathrm{T}_{1}$-weighted magnetisation-prepared rapid gradient echo sequence was used with the following parameters: inversion time: $1000 \mathrm{~ms}$, flip angle $=8$ degrees, repetition time $=2 \mathrm{~s}$, echo time $=3.37 \mathrm{~ms}$, field of view $=25.6 \mathrm{~cm}$, acquisition matrix $=256 \times 256 \times 176$, resulting in 176 contiguous sagittal slices with $1 \times 1 \times 1 \mathrm{~mm}^{3}$ isotropic spatial resolution. All scans were screened for gross radiological abnormalities by an experienced neuroradiologist. $\mathrm{N}=16$ subjects were excluded due to erroneous MR scans (2 FEP, $11 \mathrm{CHR}-\mathrm{P}$ and $3 \mathrm{HC}$ ).

MR images were processed through Freesurfer 6.0 automated segmentation pipeline (https://surfer.nmr. mgh.harvard.edu/fswiki/recon-all/). A total of 41 parcellated brain regions were obtained using the recon-all fully-automated directive workflow with the default Deskian-Killiany atlas. The workflow included motion correction, brain extraction, Talairach transformation, segmentation of cortical and subcortical structures, intensity normalization, gray matter-white matter boundary tessellation, and topology correction. Results were visually inspected and statistically evaluated for outliers following standardized ENIGMA protocols for cortical and subcortical structures (http://enigma.ini.usc.edu/protocols/imaging-protocols/) and outlier removal was performed with the code provided by the ENIGMA Consortium (http://enigma.ini.usc.edu/protocols/imaging-protocols/) and continued if the regional value was not in a range of \pm 3.5 standard deviations. Subsequently, cortical thickness, surface area and subcortical volume of all 41 brain areas were normalised with respect to intracranial volume and centred.

After the quality check of the main study population (Table 1), the full sample, with and without PSRS data, consisted of combined 22 CHR-P and 11 FEP subjects treated with antidepressants, 45 CHR-P and 57 FEP individuals without antidepressant treatment and 55 healthy controls.

Statistical analysis. The $R$ software v.3.4.0 with packages 'lmertest'v.2.0.36, 'effects' v.4.0, 'emmeans'v.0.9.1, 'car' v.2.1.6 was used for statistical, group-related descriptive analysis. Adequate statistical tests (ANOVA, logistic regression, chi-squared test or t-test) were applied to examine group effects on the following variables of interest: age, sex, handedness, years of education, IQ, BPRS, PSRS, cannabis use, antipsychotics and antidepressants (Tables 1 and S1). Additionally the Pearson correlation coefficient was calculated for BPRS ${ }^{98}$, SANS and GAF score correlated with the anatomical data of each separate region. Current mean antidepressant dose was converted into fluoxetine equivalents $^{94}$.

To investigate the medication effect on brain morphology (cortical thickness, surface area and subcortical volume), we constructed several linear mixed effect models (LME) that contained current converted antidepressant medication dose, diagnosis, sex, age, hemisphere, and interaction between current daily medication dose and hemisphere as fixed effects and intercept for every subject as random effect for each brain region. To test the hypothesis that antidepressant medication effects on cortical and subcortical structures varies in subjects with different genetic predisposition for psychosis, we constructed LME that included current converted medication dose of antidepressants, corrected PSRS score, interaction between daily medication dose and PSRS, diagnosis, sex, age, hemisphere and interaction between medication, PSRS and hemisphere as fixed effects and intercept for subject as random effect. LMEs were summarized by an ANOVA type 2 for medication effects alone (Table 2) and by an ANOVA type 3 for the interaction effects between medication and PSRS score in the PSRS subsample (Table 3).

Analyses for each region were followed by Tukey's HSD post-hoc test uncontrolled for 41 brain regions studied (Tables S6-S8, p $<0.05$ was considered significant). Likelihood ratio (LR) tests were performed for all of the constructed LME in the corresponding brain regions (Tables S4 and S5). Reduced models did not contain [medication] and [medication*hemisphere] effects in the full sample and did not contain the interactions $[P S R S *$ medication] and [PSRS*medication*hemisphere] in the PSRS subsample.

\section{References}

1. Keshavan, M. et al. A dimensional approach to the psychosis spectrum between bipolar disorder and schizophrenia: the SchizoBipolar Scale. Schizophr Res., Dec, 133(1-3), 250-4, https://doi.org/10.1016/j.schres.2011.09.005 (2011)

2. Kapur, S. Psychosis as a state of aberrant salience: a framework linking biology, phenomenology, and pharmacology in schizophrenia. Am J Psychiatry. Jan, 160(1), 13-23, https://doi.org/10.1176/appi.ajp.160.1.13 (2003)

3. McGrath, J, Saha, S, Chant, D \& Welham J. Schizophrenia: a concise overview of incidence, prevalence, and mortality Epidemiol Rev, 30 pp. 67-76, https://doi.org/10.1093/epirev/mxn001 (2008).

4. Linscott, R. J. \& van Os, J. An updated and conservative systematic review and meta-analysis of epidemiological evidence on psychotic experiences in children and adults: on the pathway from proneness to persistence to dimensional expression across mental disorders. Psychol. Med. 43, 1133-1149, https://doi.org/10.1017/s0033291712001626 (2013). 
5. Unterrassner, L. et al. Psychotic-Like Experiences at the Healthy End of the Psychosis Continuum. Front. Psychol. 8, 775, https://doi. org/10.3389/fpsyg.2017.00775 (2017).

6. Fusar-Poli, P. The Clinical High-Risk State for Psychosis (CHR-P), Version II. Schizophr Bull. Jan, 43(1), 44-47, https://doi. org/10.1093/schbul/sbw158 (2017).

7. Yung, A. R. et al. Mapping the onset of psychosis: the comprehensive assessment of at-risk mental states. Aust N Z J Psychiatry 39, 964-971, https://doi.org/10.1080/j.1440-1614.2005.01714.x (2005).

8. Borgwardt, S. J. et al. Structural brain abnormalities in individuals with an at-risk mental state who later develop psychosis. $\mathrm{Br} J$ Psychiatry Suppl. Dec, 51, 69-75, https://doi.org/10.1192/bjp.191.51.s69 (2007).

9. Fusar-Poli, P., et al. The Psychosis High-Risk State: A Comprehensive State-of-the-Art Review. JAMA Psychiatry. Jan, 70(1), 107-120, https://doi.org/10.1001/jamapsychiatry.2013.269 (2013).

10. Radua, J. et al. Ventral Striatal Activation During Reward Processing in Psychosis: A Neurofunctional Meta-Analysis. JAMA Psychiatry. Dec, 72(12), 1243-51, https://doi.org/10.1001/jamapsychiatry.2015.2196 (2015).

11. Fusar-Poli, P. et al. Disorder, not just state of risk: meta-analysis of functioning and quality of life in people at high risk of psychosis. Br J Psychiatry. Sep, 207(3), 198-206, https://doi.org/10.1192/bjp.bp.114.157115 (2015).

12. Schmidt, A. et al. Approaching a network connectivity-driven classification of the psychosis continuum: a selective review and suggestions for future research. Front Hum Neurosci. Jan, 13(8), 1047, https://doi.org/10.3389/fnhum.2014.01047 (2015).

13. Fusar-Poli, P. et al. Heterogeneity of Psychosis Risk Within Individuals at Clinical High Risk: A Meta-analytical Stratification. JAMA Psychiatry. Feb, 73(2), 113-20, https://doi.org/10.1001/jamapsychiatry.2015.2324 (2016)

14. Andreasen, N. C. et al. Progressive brain change in schizophrenia: A prospective longitudinal study of first-episode schizophrenia. Biol Psychiatry 70, 672-679, https://doi.org/10.1016/j.biopsych.2011.05.017 (2011).

15. Fusar-Poli, P. et al. Deconstructing vulnerability for psychosis: Meta-analysis of environmental risk factors for psychosis in subjects at ultra high-risk. Eur Psychiatry. Feb, 40, 65-75, https://doi.org/10.1016/j.eurpsy.2016.09.003 (2017).

16. Radua, J. et al. What causes psychosis? An umbrella review of risk and protective factors. World Psychiatry. Feb, 17(1), 49-66, https:// doi.org/10.1002/wps.20490 (2018).

17. Borgwardt, S. J. et al. Reductions in frontal, temporal and parietal volume associated with the onset of psychosis. Schizophr Res. 106, 108-114, https://doi.org/10.1016/j.schres.2008.08.007 (2008).

18. Lawrie, S. M. et al. Temporal lobe volume changes in people at high risk of schizophrenia with psychotic symptoms. Br. J. Psychiatry 181, 138-143, https://doi.org/10.1017/s0007125000161860 (2002).

19. Pantelis, C. et al. Neuroanatomical abnormalities before and after onset of psychosis: a cross-sectional and longitudinal MRI comparison. Lancet 361, 281-288, https://doi.org/10.1016/s0140-6736(03)12323-9 (2003).

20. Kasai, K. et al. Progressive decrease of left superior temporal gyrus gray matter volume in patients with first-episode schizophrenia. Am. J. Psychiatry 160, 156-164, https://doi.org/10.1176/appi.ajp.160.1.156 (2003).

21. Fornito, A. et al. Anatomic abnormalities of the anterior cingulate cortex before psychosis onset: an MRI study of ultra-high-risk individuals. Biol Psychiatry. Nov, 1, 64(9), 758-65, https://doi.org/10.1016/j.biopsych.2008.05.032 (2008)

22. Takahashi, T. et al. Insular cortex gray matter changes in individuals at ultra-high-risk of developing psychosis. Schizophr. Res, https://doi.org/10.1016/j.schres.2009.03.024 (2009).

23. Koutsouleris, N. et al. Use of neuroanatomical pattern classification to identify subjects in at-risk mental states of psychosis and predict disease transition. Arch. Gen. Psychiatry 66, 700-712, https://doi.org/10.1001/archgenpsychiatry.2009.62 (2009).

24. Smieskova, R. et al. Neuroimaging predictors of transition to psychosis-a systematic review and meta-analysis. Neurosci Biobehav Rev 34, 1207-1222, https://doi.org/10.1016/j.neubiorev.2010.01.016 (2010).

25. Koreen A. R. et al. Depression in first-episode schizophrenia. Am J Psychiatry. Nov, 150(11), 1643-8, https://doi.org/10.1016/00063223(92)90392-d (1993).

26. Bernasconi, R. et al. Hippocampal volume correlates with attenuated negative psychotic symptoms irrespective of antidepressant medication. NeuroImage Clin 8, 230-237, https://doi.org/10.1016/j.nicl.2015.04.016 (2015).

27. Mishara, L. \& Fusar-Poli P. The Phenomenology and Neurobiology of Delusion Formation During Psychosis Onset: Jaspers, Truman Symptoms, and Aberrant Salience. Schizophr Bull, Mar, 39(2), 278-86 https://doi.org/10.1093/schbul/sbs155 (2013).

28. Krabbendam, L. et al. Development of depressed mood predicts onset of psychotic disorder in individuals who report hallucinatory experiences. Br J Clin Psychol 44, 113-25, https://doi.org/10.1348/014466504x19767 (2005).

29. Falkenberg, I. et al. Why are help-seeking subjects at ultra-high risk for psychosis help-seeking? Psychiatry Res. Aug 30, 228(3), 808-15, https://doi.org/10.1016/j.psychres.2015.05.018 (2015).

30. Rutigliano, G. et al. Persistence or recurrence of non-psychotic comorbid mental disorders associated with 6-year poor functional outcomes in patients at ultra high risk for psychosis. J Affect Disord. Oct, 203, 101-110, https://doi.org/10.1016/j.jad.2016.05.053 (2016).

31. Kline E. R. et al. Depression and clinical high-risk states: Baseline presentation of depressed vs. non-depressed participants in the NAPLS-2 cohort. Schizophr Res, https://doi.org/10.1016/j.schres.2017.05.032 (2017).

32. Cornblatt, B. A. et al. Can antidepressants be used to treat the schizophrenia prodrome? Results of a prospective, naturalistic treatment study of adolescents. J. Clin. Psychiatry 68(4), 546-557, https://doi.org/10.4088/jcp.v68n0410 (2007).

33. Fusar-Poli, P., Valmaggia, L, McGuire, P. Can antidepressants prevent psychosis? Lancet. Nov 24, 370(9601), 1746-8, https://doi. org/10.1016/s0140-6736(07)61732-2 (2007).

34. Fusar-Poli, P. et al. Antidepressant, antipsychotic and psychological interventions in subjects at high clinical risk for psychosis: OASIS 6-year naturalistic study. Psychol Med. 45, 1327-1339, https://doi.org/10.1017/s003329171400244x (2015).

35. Terevnikov, V., Joffe, G. \& Stenberg, J. H. Randomized Controlled Trials of Add-On Antidepressants in Schizophrenia. Int J Neuropsychopharmacol. Jul, 18(9), https://doi.org/10.1093/ijnp/pyv049 (2015).

36. Koolschijn, P.C. et al. Brain volume abnormalities in major depressive disorder: A meta-analysis of magnetic resonance imaging studies. Human brain mapping. Nov, 30(11):3719-35, https://doi.org/10.1002/hbm.20801 (2009).

37. Tu, P. C. et al. Regional cortical thinning in patients with major depressive disorder: a surface-based morphometry study. Psychiatry Research: Neuroimaging. Jun 30, 202(3), 206-13, https://doi.org/10.1016/j.pscychresns.2011.07.011 (2012).

38. Grieve, S. M. et al. Widespread reductions in gray matter volume in depression. NeuroImage, Clinical. Jan 1, 3, 332-9, https://doi. org/10.1016/j.nicl.2013.08.016 (2013).

39. Schmaal, L. et al. Cortical abnormalities in adults and adolescents with major depression based on brain scans from 20 cohorts worldwide in the ENIGMA Major Depressive Disorder Working Group. Molecular psychiatry. Jun, 22(6), 900, https://doi. org/10.1038/mp.2016.60 (2017).

40. Smith, R. et al. Antidepressant effects of sertraline associated with volume increases in dorsolateral prefrontal cortex. Journal of Affective Disorders 146, 414-419, https://doi.org/10.1016/j.jad.2012.07.029 (2013).

41. Arnone, D. et al. State-dependent changes in hippocampal grey matter in depression. Mol Psychiatry. Dec, 18(12), 1265-72, https:// doi.org/10.1038/mp.2012.150 (2013).

42. Kong L. et al. Frontal-subcortical volumetric deficits in single episode, medication-naive depressed patients and the effects of 8 weeks fluoxetine treatment: a VBM-DARTEL study. PloS One, 9, https://doi.org/10.1371/journal.pone.0079055 (2014).

43. Modinos, G. et al. Are we really mapping psychosis risk? Neuroanatomical signature of affective disorders in subjects at ultra high risk. Psychol Med. Dec, 44(16), 3491-501, https://doi.org/10.1017/s0033291714000865 (2014).

44. Fu, C. H. Y. et al. Multimodal functional and structural neuroimaging investigation of major depressive disorder following treatment with duloxetine. BMC Psychiatry 15, 82, https://doi.org/10.1186/s12888-015-0457-2 (2015). 
45. Fossati, P., Radtchenko, A. \& Boyer, P. Neuroplasticity: from MRI to depressive symptoms. Eur. Neuropsychopharmacol. 14, 503-510, https://doi.org/10.1016/j.euroneuro.2004.09.001 (2004).

46. Schaefer, A. et al. Serotonergic modulation of intrinsic functional connectivity. Curr Biol. Oct 6, 24(19), 2314-8, https://doi. org/10.1016/j.cub.2014.08.024 (2014).

47. Richtand, N. M. \& McNamara, R. K. Serotonin and dopamine interactions in psychosis prevention. Prog Brain Res. 172, 141-53, https://doi.org/10.1016/s0079-6123(08)00907-2 (2008).

48. David, D. J. et al. Neuro genesis-dependent and -independent effects of fluoxetine in an animal model of anxiety/depression. Neuron. May 28, 62(4), 479-93, https://doi.org/10.1016/j.neuron.2009.04.017 (2009).

49. Hunsberger, J., Austin, D. R., Henter, I. D. \& Chen, G. The neurotrophic and neuroprotective effects of psychotropic agents. Dialogues Clin Neurosci 11(3), 333-48 (2009).

50. Anacker, C. et al. Antidepressants increase human hippocampal neurogenesis by activating the glucocorticoid receptor Mol Psychiatry. Jul, 16(7), 738-50, https://doi.org/10.1038/mp.2011.26 (2011).

51. Hibar et al. Cortical abnormalities in bipolar disorder: an MRI analysis of 6503 individuals from the ENIGMA Bipolar Disorder Working Group. Mol Psychiatry, https://doi.org/10.1038/mp.2017.73 (2017).

52. Huang, X. et al. Decreased left putamen and thalamus volume correlates with delusions in first-episode schizophrenia patients. Frontiers in psychiatry 8, 245, https://doi.org/10.3389/fpsyt.2017.00245 (2017)

53. Frazier, J. A. et al. Brain anatomic magnetic resonance imaging in childhood-onset schizophrenia. Archives of General Psychiatry 53.7, 617-624, https://doi.org/10.1001/archpsyc.1996.01830070065010 (1996).

54. Lawrie, S. M. et al. Brain structure, genetic liability, and psychotic symptoms in subjects at high risk of developing schizophrenia. Biological psychiatry 49.10, 811-823, https://doi.org/10.1016/s0006-3223(00)01117-3 (2001).

55. Hong, S. B. et al. Baseline putamen volume as a predictor of positive symptom reduction in patients at clinical high risk for psychosis: a preliminary study. Schizophrenia research 169(1-3), 178-185, https://doi.org/10.1016/j.schres.2015.10.029 (2015).

56. Palaniyappan, L. Progressive cortical reorganisation: a framework for investigating structural changes in schizophrenia. Neuroscience \& Biobehavioral Reviews 79, 1-13, https://doi.org/10.1016/j.neubiorev.2017.04.028 (2017).

57. Harrisberger, F. et al. Impact of polygenic schizophrenia-related risk and hippocampal volumes on the onset of psychosis. Transl. Psychiatry 6, e868, https://doi.org/10.1038/tp.2016.143 (2016).

58. Schizophrenia Working Group of the Psychiatric Genomics Consortium: Ripke et al. Biological insights from 108 schizophreniaassociated genetic loci. Nature. Jul 24, 511(7510), 421-7, https://doi.org/10.1038/nature13595 (2014)

59. Ruderfer, D. M. et al. Polygenic overlap between schizophrenia risk and antipsychotic response: a genomic medicine approach. Lancet Psychiatry. Apr, 3(4), 350-7, https://doi.org/10.1016/s2215-0366(15)00553-2 (2016).

60. Franke, B. et al. Genetic influences on schizophrenia and subcortical brain volumes: large-scale proof of concept. Nat Neurosci. Mar, 19(3), 420-431, https://doi.org/10.1038/nn.4228 (2016).

61. Terwisscha van Scheltinga, A. F. et al. Genetic schizophrenia risk variants jointly modulate total brain and white matter volume. Biol Psychiatry 73, 525-531, https://doi.org/10.1016/j.biopsych.2012.08.017 (2012).

62. Oertel-Knöchel, V et al. Schizophrenia risk variants modulate white matter volume across the psychosis spectrum: evidence from two independent cohorts. Neuroimage Clin. Mar 13, 7, 764-70, https://doi.org/10.1016/j.nicl.2015.03.005 (2015).

63. Doan, N. T. et al. Distinct multivariate brain morphological patterns and their added predictive value with cognitive and polygenic risk scores in mental disorders. Neuroimage Clin. Jun 9, 15, 719-731, https://doi.org/10.1016/j.nicl.2017.06.014 (2017).

64. Erp van, T. G. et al. Subcortical brain volume abnormalities in 2028 individuals with schizophrenia and 2540 healthy controls via the ENIGMA consortium. Mol Psychiatry. Apr, 21(4), 547-53, https://doi.org/10.1038/mp.2015.63 (2016).

65. Okada, N., et al. Abnormal asymmetries in subcortical brain volume in schizophrenia. Mol Psychiatry. Oct, 21(10), 1460-6, https:// doi.org/10.1038/mp.2015.209 (2016).

66. Farid, F, Mahadun, P. Schizophrenia-like psychosis following left putamen infarct: a case report. Journal of medical case reports. Dec, with 3(1), 7337, https://doi.org/10.4076/1752-1947-3-7337 (2009).

67. Onitsuka, T. Middle and Inferior Temporal Gyrus Gray Matter Volume Abnormalities in Chronic Schizophrenia: An MRI Study. Am J Psychiatry. Sep, 161(9), 1603-1611, https://doi.org/10.1176/appi.ajp.161.9.1603 (2004).

68. Job, D. E. et al. Grey matter changes over time in high risk subjects developing schizophrenia. Neuroimage, 25(4), https://doi. org/10.1016/j.neuroimage.2005.01.006 (2005).

69. Kuroki N. et al. Middle and Inferior Temporal Gyrus Gray Matter Volume Abnormalities in First-Episode Schizophrenia: An MRI Study. Am J Psychiatry. Dec, 163(12), 2103-2110, https://doi.org/10.1176/appi.ajp.163.12.2103 (2006).

70. Borgwardt, S. J. et al. Regional gray matter volume abnormalities in the at risk mental state. Biol Psychiatry. May 15, 61(10), 1148-56, https://doi.org/10.1016/j.biopsych.2006.08.009 (2007)

71. Meisenzahl, E. M. et al. Structural brain alterations at different stages of schizophrenia: a voxel-based morphometric study. Schizophr Res. Sep, 104(1-3), 44-60, https://doi.org/10.1016/j.schres.2008.06.023 (2008).

72. Palaniyappan, L. et al. Regional contraction of brain surface area involves three large-scale networks in schizophrenia. Schizophr Res. 129, 163-168, https://doi.org/10.1016/j.schres.2011.03.020 (2011).

73. Jalbrzikowski, M. et al. Structural abnormalities in cortical volume, thickness, and surface area in 22q11. 2 microdeletion syndrome: relationship with psychotic symptoms. NeuroImage: Clinical 3, 405-415, https://doi.org/10.1016/j.nicl.2013.09.013 (2013).

74. Rimol, L. M. et al. Cortical volume, surface area, and thickness in schizophrenia and bipolar disorder. Biological psychiatry 71.6, 552-560, https://doi.org/10.1016/j.biopsych.2011.11.026 (2012).

75. Reite, M., Teale, P., Rojas, D. C., Arciniegas, D., Sheeder, J. Bipolar disorder: anomalous brain asymmetry associated with psychosis. Am J Psychiatry. Aug, 156(8), 1159-63, https://doi.org/10.1176/ajp.156.8.1159 (1999).

76. Li, H. J. et al. Facial emotion processing in patients with schizophrenia and their non-psychotic siblings: a functional magnetic resonance imaging study. Schizophr Res. 134(2-3), 143-50, https://doi.org/10.1016/j.schres.2011.10.019 (2011).

77. Job, E. D. et al. Structural Gray Matter Differences between First-Episode Schizophrenics and Normal Controls Using Voxel-Based Morphometry. NeuroImage 17(2), 880-889, https://doi.org/10.1016/s1053-8119(02)91180-8 (2002).

78. Morgan KD et al. Grey matter abnormalities in first-episode schizophrenia and affective psychosis. Br J Psychiatry Suppl. Dec, 51:111-6, https://doi.org/10.1192/bjp.191.51.s111 (2007).

79. Diederen K. et al. Decreased language lateralization is characteristic of psychosis, not auditory hallucinations. Brain Dec; 13312 , 3734-3744, https://doi.org/10.1093/brain/awq313 (2010)

80. Hatton, S. N. et al. Cortical thinning in young psychosis and bipolar patients correlate with common neurocognitive deficits. International Journal of Bipolar Disorders 1(1), 1-13, https://doi.org/10.1186/2194-7511-1-3 (2013).

81. Mason, L., Peters, E., Williams, S. C., Kumari, V. Brain connectivity changes occurring following cognitive behavioural therapy for psychosis predict long-term recovery. Transl. Psychiatry. Jan 17, 7(1), e1001, https://doi.org/10.1038/tp.2017.194 (2017).

82. Hogstrom, L. J., Westlye, L. T., Walhovd, K. B. \& Fjell, A. M. The structure of the cerebral cortex across adult life: age-related patterns of surface area, thickness, and gyrification. Cereb Cortex 23, 2521-2530, https://doi.org/10.1093/cercor/bhs231 (2013).

83. Panizzon, M. S. et al. Distinct genetic influences on cortical surface area and cortical thickness. Cereb Cortex 19, 2728-2735, https:// doi.org/10.1093/cercor/bhp026 (2009).

84. Vernon, J. A. et al. Antidepressants for cognitive impairment in schizophrenia-a systematic review and meta-analysis. Schizophr Res. Nov, 159(2-3), 385-94, https://doi.org/10.1016/j.schres.2014.08.015 (2014). 
85. Helfer, B. et al. Efficacy and safety of antidepressants added to antipsychotics for schizophrenia: a systematic review and metaanalysis. Am J Psychiatry, https://doi.org/10.1176/appi.ajp.2016.15081035 (2016)

86. Phillips, J. L., Batten, L. A., Tremblay, P., Aldosary, F. \& Blier P. A prospective, longitudinal study of the effect of remission on cortical thickness and hippocampal volume in patients with treatment-resistant depression. International Journal of neuropsychopharmacology. Jun 1, 18(8), https://doi.org/10.1093/ijnp/pyv037 (2015).

87. Nelson, B. et al in Early Psychosis: a sequential multiple assignment randomised trial of interventions for ultra high risk of psychosis patients. Early intervention in psychiatry. Jun, 12(3), 292-306, https://doi.org/10.1111/eip.12459 (2017).

88. Rapp, C., Bugra H., Riecher-Rossler, A., Tamagni, C., Borgwardt, S. Effects of cannabis use on human brain structure in psychosis: a systematic review combining in vivo structural neuroimaging and post mortem studies. Current pharmaceutical design. Nov 1, 18(32), 5070-80, https://doi.org/10.2174/138161212802884861 (2012).

89. Gillespie, N. A. et al. Testing associations between cannabis use and subcortical volumes in two large population-based samples. Addiction 113.9, 1661-1672, https://doi.org/10.1111/add.14252 (2018).

90. Moreno-Alcázar, A. et al. Larger grey matter volume in the basal ganglia of heavy cannabis users detected by voxel-based morphometry and subcortical volumetric analysis. Frontiers in psychiatry 9, 175, https://doi.org/10.3389/fpsyt.2018.00175 (2018).

91. Weiland, B. et al. Daily marijuana use is not associated with brain morphometric measures in adolescents or adults. Journal of Neuroscience 35.4, 1505-1512, https://doi.org/10.1523/jneurosci.2946-14.2015 (2015).

92. Riecher-Rössler, A. et al. The Basel early-detection-of-psychosis (FEPSY)-study-design and preliminary results. Acta Psychiatr Scand. Feb, 115(2), 114-25, https://doi.org/10.1111/j.1600-0447.2006.00854.x (2007).

93. Yung, A. R. et al. Monitoring and care of young people at incipient risk of psychosis. Schizophr Bull. 22(2), 283-303, https://doi. org/10.1176/foc.2.1.158 (1996).

94. Hayasaka, Y. et al. Dose equivalents of antidepressants: evidence-based recommendations from randomized controlled trials. J Affect Disord. 180, 179-184, https://doi.org/10.1016/j.jad.2015.03.021 (2015).

95. Ho, B. C., Andreasen, N. C., Ziebell, S., Pierson, R., Magnotta, V. Long-term antipsychotic treatment and brain volumes: a longitudinal study of first-episode schizophrenia. Archives of general psychiatry. Feb 7, 68(2), 128-37, https://doi.org/10.1001/ archgenpsychiatry.2010.199 (2011).

96. Wray, N. R. et al. Research review: polygenic methods and their application to psychiatric traits. J Child Psychol Psychiatry 55, 1068-1087, https://doi.org/10.1111/jcpp.12295 (2014).

97. International Schizophrenia Consortium, Purcell, S. M. et al. Common polygenic variation contributes to risk of schizophrenia and bipolar disorder. Nature 460, 748-752, https://doi.org/10.1038/nature08185 (2009).

98. Shafer, A. et al. Factor Structure of the Brief Psychiatric Rating Scale - Expanded (BPRS-E) in a Large Hospitalized Sample. J Psychiatr Res 93, 79-86, https://doi.org/10.1016/j.jpsychires.2017.05.011 (2017).

\section{Acknowledgements}

We thank out radiographer, Tanja Haas, from Basel University Hospital for performing the magnetic resonance measurements and the FePsy Study Group for the recruitment and management of the study participants. We thank all participants for taking part in our study.

\section{Author Contributions}

F.H. and S.B. conceived and designed the study. Frueherkennung von Psychosen Study Group recruited and managed the participants. F.H. and L.E. performed the MRI scans. F.H. collected and analysed genetics data. O.B. participated in the design, did the MRI processing, analysed data and wrote the manuscript with the substantial help from F.H., A.S., D.H. and S.B. All authors reviewed the manuscript.

\section{Additional Information}

Supplementary information accompanies this paper at https://doi.org/10.1038/s41598-019-44903-y.

Competing Interests: The authors declare no competing interests.

Publisher's note: Springer Nature remains neutral with regard to jurisdictional claims in published maps and institutional affiliations.

(c) (1) Open Access This article is licensed under a Creative Commons Attribution 4.0 International

License, which permits use, sharing, adaptation, distribution and reproduction in any medium or format, as long as you give appropriate credit to the original author(s) and the source, provide a link to the Creative Commons license, and indicate if changes were made. The images or other third party material in this article are included in the article's Creative Commons license, unless indicated otherwise in a credit line to the material. If material is not included in the article's Creative Commons license and your intended use is not permitted by statutory regulation or exceeds the permitted use, you will need to obtain permission directly from the copyright holder. To view a copy of this license, visit http://creativecommons.org/licenses/by/4.0/.

(c) The Author(s) 2019 